\title{
Subharmonic Structures of Single-Electron Tunneling
}

\author{
C. D. $\mathrm{HU}$ \\ Department of Physics, National Taiwan University, \\ Taipei, Taiwan, Republic of China
}

(Received November 11, 1994)

\begin{abstract}
We used the Bloch theory to study single-electron tunneling. It was found that calculation can be performed easily in the crystal momentum representation. We showed analytically that under ac-biased voltage or microwave radiation the system produces response with subharmonic structures analogous to those of Josephson tunneling. Our method treated the "interband transition" as small perturbation and thus fits most experimental conditions. Some numerical results were also given.
\end{abstract}

\section{§1. Introduction}

In the past ten years much effort has been devoted to single-electron tunneling (SET). ${ }^{1)}$ Usually the system consists of two metallic electrodes and an island in the middle. They are seperated by insulating gaps through which electrons can tunnel. If the island is sufficiently small, the charging energy will be significant due to its low capacitance. The island becomes a Coulomb blockade and produces such interesting physical phenomena as phase-charge duality, co-tunneling, the electron turnstile and pump and quantum electrodynamic circuits. ${ }^{2)}$ Now junction arrays can be fabricated and new interesting properties were found. SET is also closely related to physical systems such as single-electron junctions, quantum dots and wires, resonant tunneling structures and thin metallic rings. ${ }^{3)}$

Theoretical methods applied to SET range from semi-classical approach of calculating hopping probability ${ }^{4}$ to path-integral adapted from single-electron tunneling. ${ }^{5)}$ Averin and Likhrarev ${ }^{6}$ used the Bloch theory to give a clear picture to this problem. They obtained a master equation and calculated the dc I-V curve numerically. They found subharmonic phase-locking results when the system was under ac current bias. $\mathrm{Hu}$ and $\mathrm{O}^{\prime}$ Connell' studied the Josephson tunneling using the Langevin equation. They gave quantitative analysis of dc current and I-V characteristics. However, as they discussed in details, Bloch osillation is different from the Josephson oscil- lation. Subharmonic structures had been found in single-electron junctions irradiated by microwave. ${ }^{8)}$ Hasselberg et al. ${ }^{9)}$ proposed two theories, multi-particle tunneling (mpt) and self-coupling (sc), to explain this phenomenon. However, both have limitations. In this work we proposed to use the Bloch theory study SET. It is physically transparent in the energy-band picture and has wider range of applicability. I-V curves can be easily calculated. Subharmonic structures can be studied analytically. This method is also relevant to related problems stated above. The theory is presented in $\$ 2$. We give some numerical results in $\$ 3$ and conclusion in $\S 4$.

\section{§2. Model Hamiltonian}

For single-electron tunneling through a junction or an island in between, the Hamiltonian is

$$
H_{0}=-\frac{(e \hat{N})^{2}}{2 C}+H_{\mathrm{T}}
$$

where $C$ is the capacitance of the junction or the island and $\hat{N}$ is the number operator of electrons. The expectation value of $\hat{N}$ in the island or at the junctions is not necessary an integer. $\hat{N}$ does not commute with the Hamiltonian due to the presence of $H_{\mathrm{T}} . H_{\mathrm{T}}$ is composed of two parts, tunneling to the left and right electrode, or into and out of the island:

$$
H_{\mathrm{T}}=H_{+}+H_{-} \text {. }
$$

Since ${ }^{6}$ 


$$
\left[\hat{N}, H_{ \pm}\right]= \pm H_{ \pm}
$$

we can introduce the phase operator $\phi$ which is canonically conjugate to $\hat{N}$ :

$$
\hat{N}=-i \frac{\partial}{\partial \phi} \text {. }
$$

such that

$$
H_{ \pm}=\frac{1}{2} E_{T} e^{ \pm i \phi}
$$

where $E_{\mathrm{T}}$ is the tunneling energy. $H_{ \pm}$satisfies the commutation relation of (3). Therefore, the original Hamiltonian can be written as

$$
H_{0}=-\frac{e^{2}}{2 C} \frac{\partial^{2}}{\partial \phi^{2}}+E_{T} \cos \phi
$$

in the $\phi$ representation. When the system is under ac-voltage bias or irradiated by microwaves, the total Hamiltonian is

$$
H=H_{0}+e\left[V_{0}+V_{1} \sin (\omega t+\alpha)\right] \hat{N}
$$

where $\alpha$ is a constant phase and $V_{0}$ and $V_{1}$ are $\mathrm{dc}$ and ac voltages respectively. It gives the well-known equation of motion:

$$
\ddot{\phi}=\frac{e^{2} E_{T}}{C} \sin \phi+V_{1} \omega \cos (\omega t+\alpha) .
$$

The conventional way of solving this problem is assuming the system varies adiabatically with the ac bias voltage. Using the relation

$$
\dot{\phi}=e V(t)
$$

where

$$
V(t)=V_{0}+V_{1} \sin (\omega t+\alpha)
$$

and substituting $\phi$ into (5), one is able to obtain ac current response at higher harmonics. In this article we present a fully quantum mechanical treatment.

We use an approach similar to that proposed by Shirley. ${ }^{11)}$ According to Flouquet's theorem the solutions of the Schrödinger equation involving $H_{0}$ only have the form $e^{i k \phi} u_{k}(\phi)$ where $u_{k}(\phi)$ is periodic in $\phi$. In the energy band language, $k$ is the crystal momentum. The period of "potential" is $2 \pi$ and the "reciprocal lattice vectors" are integers. There is no band index because we found that the extended zone scheme is the most suitable for our purpose. Consider the case $V \ll e^{2} / 2 C$. The wave function is approximately $e^{i k \phi}$. An interband transition to nearest bands which increases or decreases $k$ by unity corresponds to tunneling of one electron. In view of eq. (4), this gives the correct value of $N$. Thus, we present calculation in the extended zone scheme which not only is physically more transparent but also leaves no ambiguity in the phase of $u_{k}(\phi)$. In the "crystal momentum representation" (CMR) with $\phi$ corresponding to the spatial coordinates, we found, in the matrix form,

$$
[\mathbf{H}(t)]_{p q}=\delta_{p, q}\left\{\varepsilon_{p}+e\left(p+\mathbf{P}_{p p}\right)\left[V_{0}+V_{1} \sin (\omega t+\alpha)\right]\right\}+\sum_{n} \delta_{p, n+q} e\left[V_{0}+V_{1} \sin (\omega t+\alpha)\right] \mathbf{P}_{p q}
$$

where $\varepsilon_{p}$ is the eigenenergy of $H_{0}$ and

$$
\mathbf{P}_{p q}=-i \int e^{i(q-p) \phi} u_{p}^{*}(\phi) \frac{\partial}{\partial \phi} u_{q}(\phi) d \phi .
$$

In the reduced zone scheme usually used, $\delta_{p, n+q}$ where $n$ is a nonzero integer gives a vertical interband transition and $\boldsymbol{H}(t)$ is diagonal in the "crystal momentum". ${ }^{12)}$ Since the interband transition matrix $\boldsymbol{P}$ usually is very small we can treat it as a perturbation. So, by regrouping terms, we have

$$
H=H_{1}+H_{2}
$$

where

$$
\begin{aligned}
& {\left[\mathbf{H}_{1}\right]_{p q}=\delta_{p, q}\left[\varepsilon_{q}+e \bar{p} V_{0}+e \bar{p} V_{1} \sin (\omega t+\alpha)\right],} \\
& {\left[\mathbf{H}_{2}\right]_{p q}=\sum_{n} \delta_{p, n+q} e\left[V_{0}+V_{1} \sin (\omega t+\alpha)\right] \mathbf{P}_{p q}}
\end{aligned}
$$

and 


$$
\bar{p}=p+\mathbf{P}_{p p}
$$

In the interaction picture

$$
i \hbar \frac{\partial}{\partial t} \mathbf{U}(t)=\mathbf{H}_{2, I}(t) \mathbf{U}(t)
$$

where

$$
\begin{aligned}
{\left[\mathbf{H}_{2, I}(t)\right]_{p q}=} & \exp \left\{\operatorname{it}\left[\varepsilon_{p q}+e V_{0}(\bar{p}-\bar{q})\right] / \hbar+i e V_{1}(\bar{q}-\bar{p})[\cos (\omega t+\alpha)-\cos \alpha] / \hbar \omega\right\} \\
& \times \sum_{n} \delta_{p, n+q} e\left[V_{0}+V_{1} \sin (\omega t+\alpha)\right] \mathbf{P}_{p q}
\end{aligned}
$$

with $\varepsilon_{p q}=\varepsilon_{p}-\varepsilon_{q}$. The exponential factor comes from the intraband time-evolution. We found

$$
\begin{aligned}
{[\mathbf{U}(t)]_{p q}=} & \delta_{p q}-\frac{i}{\hbar} \int_{0}^{t} \mathrm{~d} t^{\prime} \sum_{k, n} \delta_{p, n+k} \\
& \left.\times \exp \left\{i t^{\prime}\left[\varepsilon_{p q}+e V_{0}(\bar{p}-\bar{k})\right] / \hbar+i e V_{1}(\bar{k}-\bar{p}) \cos \left(\omega t^{\prime}+\alpha\right)-\cos \alpha\right] / \hbar \omega\right\} \\
& \times e\left[V_{0}+V_{1} \sin \left(\omega t^{\prime}+\alpha\right)\right] \mathbf{P}_{p k} \mathbf{U}_{k q}\left(t^{\prime}\right) .
\end{aligned}
$$

The term which is first order in $\boldsymbol{P}$ is

$$
\begin{aligned}
{[\mathbf{U}(t)]_{p q}^{(1)}=} & \sum_{n} \delta_{p, q+n} \mathbf{P}_{p q} \exp \left\{(i t / \hbar)\left[\varepsilon_{p q}+e V_{0}(\bar{p}-\bar{q})\right]\right\} \\
& \times\left[J_{0}\left(\frac{e V_{1}(\bar{q}-\bar{p})}{\hbar \omega}\right) \frac{e V_{0}}{\varepsilon_{p p}+e V_{0}(\bar{q}-\bar{p})}+2 \sum_{m} i^{m} J_{m}\left(\frac{e V_{1}(\bar{q}-\bar{p})}{\hbar \omega}\right)\right. \\
& \times\left(\frac{e V_{0}(\bar{q}-\bar{p}) \varepsilon_{p p}+\left[e V_{0}(\bar{q}-\bar{p})\right]^{2}}{\left[\varepsilon_{q p}+e V_{0}(\bar{q}-\bar{p})\right]^{2}-(m \hbar \omega)^{2}} \cos (m \omega t+m \alpha)\right. \\
& \left.\left.-\frac{i m \hbar \omega \varepsilon_{q p}}{\left[\varepsilon_{q p}+e V_{0}(\bar{q}-\bar{p})\right]^{2}-(m \hbar \omega)^{2}} \sin (m \omega t+m \alpha)\right)\right] \\
& -\sum_{n} \delta_{p, q+n} \mathbf{P}_{p q}\left[J_{0}\left(\frac{e V_{1}(\bar{q}-\bar{p})}{\hbar \omega}\right) \frac{e V_{0}}{\varepsilon_{q p}+e V_{0}(\bar{q}-\bar{p})}\right. \\
& +2 \sum_{m} i^{m} J_{m}\left(\frac{e V_{1}(\bar{q}-\bar{p})}{\hbar \omega}\right)\left(\frac{e V_{0}(\bar{q}-\bar{p}) \varepsilon_{q p}+\left[e V_{0}(\bar{q}-\bar{p})\right]^{2}}{\left[\varepsilon_{q p}+e V_{0}(\bar{q}-\bar{p})\right]^{2}-(m \hbar \omega)^{2}} \cos m \alpha\right. \\
& \left.\left.-\frac{i m \hbar \omega \varepsilon_{q p}}{\left[\varepsilon_{q p}+e V_{0}(\bar{q}-\bar{p})\right]^{2}-(m \hbar \omega)^{2}} \sin m \alpha\right)\right]
\end{aligned}
$$

where we used the relation

$$
\exp (i z \cos \phi)=J_{0}(z)+2 \sum_{n} i^{n} J_{n}(z) \cos n \phi .
$$

With the time-evolution matrix known, we are able to calculate expectation values of physical observables.

It is interesting to analysis the I-V characteristics. The measured current is

$$
I(t)=e\langle\dot{N}\rangle=\frac{E_{T}}{\hbar}\langle\sin \phi(t)\rangle .
$$

Using the time evolution operator we have

$$
I(t)=\frac{E_{T}}{\hbar}\left\langle\mathbf{U}^{\dagger}(t) \exp \left[i / \hbar \int_{0}^{t} \mathbf{H}_{1}\left(t^{\prime}\right) d t^{\prime}\right] \sin \phi \exp \left[-i / \hbar \int_{0}^{t} \mathbf{H}_{1}\left(t^{\prime}\right) \mathrm{d} t^{\prime}\right] \mathbf{U}(t)\right\rangle .
$$


Consider only the first order interband transition

$$
\begin{aligned}
I_{p p}(t)= & \frac{E_{T}}{\hbar}\left(\left\langle[\mathbf{U}]^{(1)^{\dagger}}(t) \exp \left[i / \hbar \int_{0}^{t} \mathbf{H}_{1}\left(t^{\prime}\right) \mathrm{d} t^{\prime}\right] \sin \phi \exp \left[-i / \hbar \int_{0}^{t} \mathbf{H}_{1}\left(t^{\prime}\right) \mathrm{d} t^{\prime}\right]\right\rangle_{p p}\right. \\
& \left.+\left\langle\exp \left[i / \hbar \int_{0}^{t} \mathbf{H}_{1}\left(t^{\prime}\right) \mathrm{d} t^{\prime}\right] \sin \phi \exp \left[-i / \hbar \int_{0}^{t} \mathbf{H}_{1}\left(t^{\prime}\right) \mathrm{d} t^{\prime}\right][\mathbf{U}]^{(1)}(t)\right\rangle_{p p}\right) \\
= & \sum_{n, q} \frac{E_{T}}{\hbar} \delta_{p, n+q}\langle\sin \phi\rangle_{p q} \exp \left[i e V_{1}(\bar{p}-\bar{q}) \cos \alpha / \hbar \omega\right] \\
& \times\left[J_{0}\left(\frac{e V_{1}(\bar{q}-\bar{p})}{\hbar \omega}\right)+2 \sum_{l} i^{l} J_{l}\left(\frac{e V_{1}(\bar{q}-\bar{P})}{\hbar \omega}\right) \cos (l \omega t+l \alpha)\right] \mathbf{P}_{q p} \\
& \times\left[J_{0}\left(\frac{e V_{1}(\bar{q}-\bar{p})}{\hbar \omega}\right) \frac{e V_{0}}{\varepsilon_{q p}+e V_{0}(\bar{q}-\bar{p})}+2 \sum_{m} i^{m} J_{m}\left(\frac{e V_{1}(\bar{q}-\bar{p})}{\hbar \omega}\right)\right. \\
& \times\left(\frac{e V_{0}(\bar{q}-\bar{p}) \varepsilon_{q p}+\left[e V_{0}(\bar{q}-\bar{p})\right]^{2}}{\left[\varepsilon_{q p}+e V_{0}(\bar{q}-\bar{p})\right]^{2}-(m \hbar \omega)^{2}} \cos (m \omega t+m \alpha)\right. \\
& \left.\left.-\frac{i m \hbar \omega \varepsilon_{q p}}{\left[\varepsilon_{q p}+e V_{0}(\bar{q}-\bar{p})\right]^{2}-(m \hbar \omega)^{2}} \sin (m \omega t+m \alpha)\right)\right]+c . c .
\end{aligned}
$$

where eqs. (11), (16) and (17) were used. The dc part of the current can be extracted by collecting the terms with $l=m$. For a given $n$,

$$
\begin{aligned}
I_{p p}^{\mathrm{dc}, n}= & \frac{E_{T}}{\hbar} \cos \left[\bar{n} e V_{1}(\cos \alpha) / \hbar \omega\right]\langle\sin \phi\rangle_{p, p+n} \mathbf{P}_{p+n, p} \\
& \times\left[J_{0}^{2}\left(\frac{\bar{n} e V_{1}}{\hbar \omega}\right) \frac{e V_{0}}{\varepsilon_{p+n, p}+\bar{n} e V_{0}}+2 \sum_{m}(-1)^{m} J_{m}^{2}\left(\frac{\bar{n} e V_{1}}{\hbar \omega}\right) \frac{2 \bar{n} e V_{0} \varepsilon_{p+n, p}+\left(\bar{n} e V_{0}\right)^{2}}{\left[\varepsilon_{p+n, p}+\bar{n} e V_{0}\right]^{2}-(m \hbar \omega)^{2}}\right]
\end{aligned}
$$

where we have used the Hermitian property of $\boldsymbol{P}$. In eq. (23), we found that the dc current is the largest when $-e V_{0}=\left(\varepsilon_{p+n, p} \pm m \hbar \omega\right) / \bar{n}$. Dissipation which always exists should remove the divergence. Its magnitude is proportional to $J_{m}^{2}\left(\bar{n} e V_{1} / \hbar \omega\right)$. Due to the arbitrariness of $\alpha$ the dc current has a steplike line shape. Note that in the argument of Bessel functions $\bar{n}$ is not an integer. However, it is very close to $n$ even for fairly large $E_{\mathrm{T}}$ (see Table II and explanation in next section). Experimentally the difference probably is not distinquishable. It is well-known that Josephson tunneling irradiated by microwaves exhibits subharmonic structures at $e V_{0}=(2 \Delta+m \hbar \omega) / n$ where $\Delta$ is the energy gap. The dc current magnitude is proportional to $J_{m}^{2}\left(n e V_{1} / \hbar \omega\right)$. This is very similar to the result in (24). Therefore we concluded that subharmonic structures phenomenon is not restricted to Josephson tunneling and it had the same origin in both single-electron tunneling and Josephson tunneling.
Hasselberg et al. ${ }^{9)}$ proposed two theories for the Josephson tunneling. The multiple-particle tunneling (mpt) theory though neglected the self-induced voltage gave dc current steps at right places and correct magnitudes. However, it failed to produce some fine line shapes. The self-coupling (sc) theory provided the detailed structures but it could only give odd- $n$ ( $m$ in ref. 8 ) series of steps (quasi-particles) and peaks (cooper pairs). Pair-breaking mechanism has to be invoked to produce the even- $n$ series. It has to be pointed out that both theories treated the tunneling part as a small perturbation. Our theory does not have above limitations. We took only the interband transition part as perturbation. The self-induced (or tunneling-induced) voltage, which corresponds to the the potential influence on the Bloch electron velocity in CMR, has been taken into account. To evaluate dc current we can easily calculate the "energy bands" which includes the contribution of "potential 
energy" or the tunneling term. Even the applied voltage contribution to self-coupling was considered partially in our time-evolution operator $\exp \left[-i / \hbar \int_{0}^{t} \boldsymbol{H}_{1}(t) \mathrm{d} t\right] \boldsymbol{U}^{(1)}(t)$. Thus, our theory can be used to describe Josephson tunneling with some modification.

\section{§3. Numerical Results}

In this section we give some numerical values in order to have some feeling of the entire process. They were evaluated with the method of continual fraction. ${ }^{10,13)}$ The "energy bands" are shown in Fig. 1. They were evaluated under the condition $V=e^{2} / 2 C$ where treating tunneling as perturbation begins to fail. The energy unit is $e^{2} / 2 C$. The only feature is the energy gaps at Brillouin zone boundaries. The wave functions are also calculated. We have

$$
u_{k}(\phi)=\sum_{m} a_{m} \mathrm{e}^{\mathrm{i} m \phi} .
$$

The most important $a$ 's of $u_{k}(\phi)$ for typical $k$ values: $0.1,1.1,2.1$ were listed in Table $I$. With $a$ 's known, we evaluated the matrix elements of $\boldsymbol{P}$ and $\langle\sin \phi\rangle / i$. Those between above $k$ values were listed in Table II and III respectively. We found that for $\boldsymbol{P}$ the transitions between nearest bands have largest magnitudes. As for $\langle\sin \phi\rangle / i$, the matrix elements

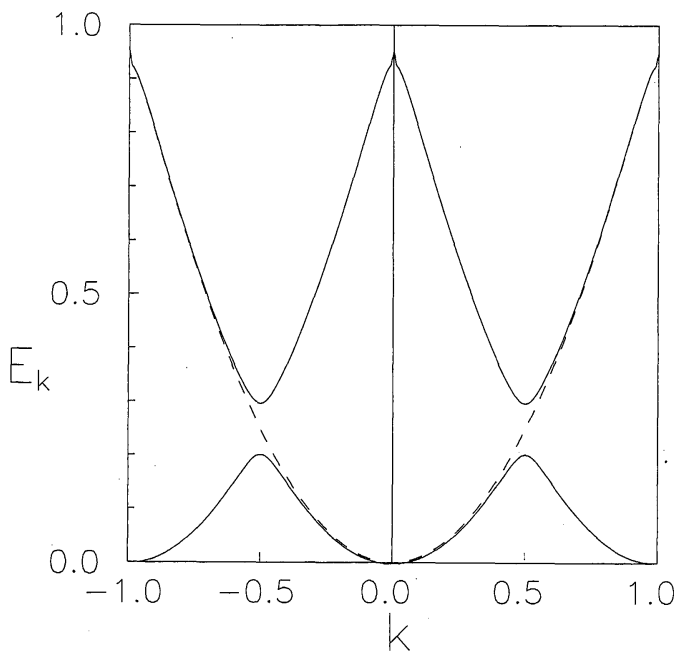

Fig. 1. "Energy bands" of $H_{0}$ under the condition of $E_{\mathrm{T}}=e^{2} / 2 C$. The dashed line shows the energy of free particles $\left(E_{\mathrm{T}}=0\right)$. The energy unit is $e^{2} / 2 C$.
Table I. Normalized fourier components of $u_{k}(\phi)$ times 1000 where $u_{k}(\phi)=\Sigma_{m} a_{m} e^{i m \phi}$.

\begin{tabular}{ccrccl}
\hline$k$ & $a_{-2}$ & \multicolumn{1}{c}{$a_{-1}$} & $a_{0}$ & \multicolumn{1}{c}{$a_{1}$} & \multicolumn{1}{c}{$a_{2}$} \\
\hline 0.1 & 2.042 & -59.30 & 392.5 & -39.95 & 1.127 \\
1.1 & 12.74 & 42.30 & 396.2 & -15.53 & 0.2313 \\
2.1 & 0.4427 & 15.58 & 398.5 & -9.586 & 0.09665 \\
\hline
\end{tabular}

Table II. Matrix elements of $\boldsymbol{P}$. The first column and first row indicate the values of "crystal momentum".

\begin{tabular}{cccc}
\hline$k$ & 0.1 & \multicolumn{1}{c}{1.1} & \multicolumn{1}{c}{2.1} \\
\hline 0.1 & -0.00203 & -0.0949 & 0.00174 \\
1.1 & -0.0949 & -0.00187 & -0.0390 \\
2.1 & 0.00174 & -0.0390 & -0.00015 \\
\hline
\end{tabular}

Table III. Matrix elements of $\langle\sin \phi\rangle / i$. The first column and first row indicate the values of "crystal momentum”. Diagonal matrix elements vanish.

\begin{tabular}{cccc}
\hline$k$ & 0.1 & 1.1 & 2.1 \\
\hline 0.1 & 0 & 0.471 & -0.0309 \\
1.1 & -0.471 & 0 & 0.498 \\
2.1 & 0.0309 & -0.498 & 0 \\
\hline
\end{tabular}

between nearest bands are also largest while the diagonal elements vanish. This can be easily understood physically. Nearest bands can be explained approximately as differing in $N$ by one. The current is the greatest while transferring only one electron. The matrix elements of $\boldsymbol{P}$ are always smaller than one. Our perturbation procedure is valid for most situations.

Equation (25) shows an interesting relation between current and frequency. We show it in Figs. 2 and 3 . Only the case $n=1$ was considered because otherwise the current would be negligible. Since $\alpha$ varies with different charging condition, the factor $\cos \left[\bar{n} e V_{1}(\cos \alpha) /\right.$ $\hbar \omega]$ will be averaged out. The currents shown in Figs. 2 and 3 are those avereaged over all $p$ states. A dissipation constant was introduced to remove the divergence at $-e V_{0}=$ $\left(\varepsilon_{p+n, p} \pm m \hbar \omega\right) / \bar{n}$. Its net effect is to replace $\omega$ with $\omega-i \eta$. It could comes from the thermal fluctuation. Figures 2 and 3 were plotted under the condition $E_{\mathrm{T}}=e^{2} / 2 C=V_{0}=100 \eta$. The solid line is the current when $V_{1}=0.25 V_{0}$ and the dashed line is that when $V_{1}=0.15 V_{0}$. The currents are in arbitrary unit and the fre- 


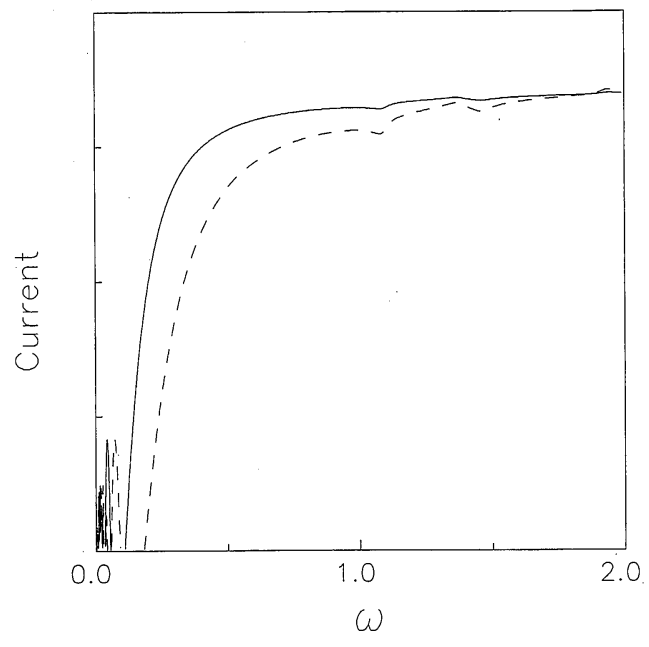

Fig. 2. Current (in arbitrary unit) as a function of frequency $\omega$ in the unit of $V_{0}$. We plotted the cases of $E_{\mathrm{T}}=e^{2} / 2 C=V_{0}$ and $V_{1}=0.25 V_{0}$ for the solid lins and $V_{1}=0.15 V_{0}$ for the dashed line.

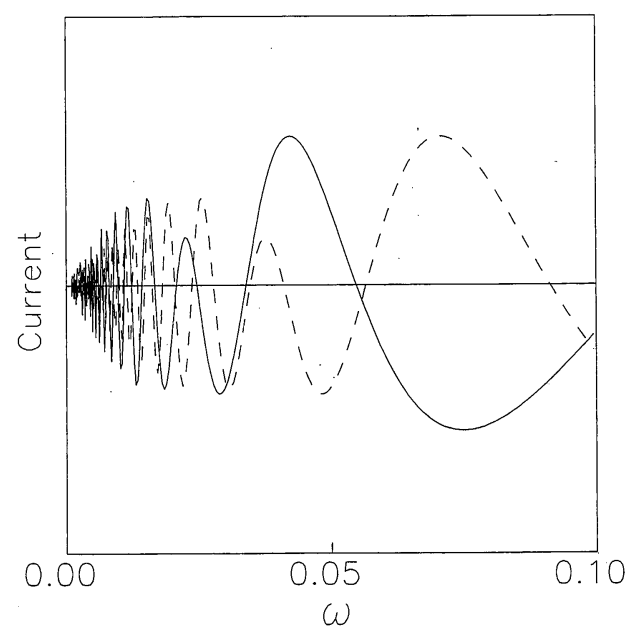

Fig. 3. Shows the details in small frequencies with all the conditions the same as those in Fig. 2.

quency is in the unit of $V_{0}$. For large frequency, the current approaches a constant because the only non-vanishing term is that of $J_{0}\left(e V_{1} / \hbar \omega\right)$. There are oscllations in small frequency shown in Fig. 3. The peaks occur near the zeros of $J_{1}\left(e V_{1} / \hbar \omega\right)$ and the valleys occur near the zeros of $J_{0}\left(e V_{1} / \hbar \omega\right)$. All other higher order Bessel functions give contributions dominated by those of the first two.

\section{§4. Conclusion}

The well-developed enegy-band theory can be applied to SET. Starting from $H_{0}$, we built up energy bands. Under a time-varying external field, the "intraband" part can be diagonalized. The "interband transition" is treated as perturbation. This way we incorporated the tunneling Hamiltonian which gives self-coupling voltage to the tunnel junction. In CMR, the properties of wave functions are well-known. Various calculations are also straightforward. "Interband transition"' were shown to be small. We derived analytically the dc current response. It has a line shape of steps occuring at subharmonic frequencies. The magnitude is proportional to $J_{m}^{2}\left(\bar{n} e V_{1} /\right.$ $\hbar \omega)$. All features are very similar to those of the subharmonic structures in the single-electron tunneling except that we did not consider pair breaking.

There is remarkable analogy between singleelectron tunneling, a Bloch electron and a quantum pendulum. The charging energy plays the role of kinetic energy. The tunneling energy corresponds to the potential for the Bloch electron and the source of the nonlinear restoring force for the pendulum. The ac voltage bias or interaction with microwave is a time-dependent field. Different bands correspond approximately to different charging states. "Interband transition" is similar to transferring charge. Therefore, CMR can readily be applied to SET. It has the additional advantange of taking account of the nonlinear force almost exactly. Furthermore, due to the similarity of single-electron tunneling, to quantum dots and wires and thin rings, we believe that CMR can also be applied to these problems.

This work is supported in part by the NSC of Taiwan, R.O.C. under the contract number NSC84-2112-M-002-012.

\section{References}

1) For recent reviews, see Single Charge Tunneling, ed. H. Grabert and M. Devoret, NATO ASI Series B: Physics (Plenum Press, New York, 1992) Vol. 294; H. Koch and H. Lubbig: Single-Electron Tunneling and Mesoscopic Device (Springer-Verlag, Berlin, New York, 1992).

2) A. Widom, G. Megaloudis, T. D. Clark, H. Prance 
and R. J. Prance: J. Phys. A15 (1982) 3887.

3) See for example, N. Byers and C. N. Yang: Phys. Rev. Lett. 7 (1961) 46; Y. Gefen, Y. Imery and M. Ya Azbel: Phys. Rev. Lett. 53 (1984) 129; M. Buttiker: Phys. Rev. B32 (1985) 1846; E. Ben-Jacob, Y. Gefen, K. Mullen and Z. Schuss: Phys. Rev. B37 (1988) 7400; D. Takai and K. Ohta: Phys. Rev. B48 (1993) 14318.

4) The fourth article of ref. 3 .

5) G. Schön and A. D. Zaikin: Phys. Rep. 198 (1990) 137.

6) D. V. Averin and K. K. Likharev: J. Low Temp. Phys. 62 (1986) 345.

7) G. Y. Hu and R. F. O'Connell: Phys. Rev. B47
(1993) 8823.

8) I. Giaever and H. R. Zeller: Phys. Rev. B1 (1970) 4278.

9) L.-E. Hasselberg, M. T. Levinsen and M. R. Samuelsen: Phys. Rev. B9 (1974) 3757.

10) The author thanks J. Mahanty for pointing this out to him.

11) J. H. Shirley: Phys. Rev. 138 (1965) B979.

12) E. I. Blount: Formalisms of Band Theory, ed. F. Seitz and D. Turnbull, Solid State Phys. (Academic Press, New York, London, 1962) Vol. 13.

13) P. M. Morse and H. Feshbach: Methods of Theoretical Physics (McGraw-Hill, New York, 1978). 